\title{
Establishing a Molecular COMMUNiCATion CHANNEL FOR NANO NETWORKS
}

\author{
Prachi Raut ${ }^{1}$ and Nisha Sarwade ${ }^{2}$ \\ ${ }^{1}$ Department of Electrical Engineering, VJTI, Mumbai, India \\ rautps@gmail.com \\ ${ }^{2}$ Department of Electrical Engineering, VJTI, Mumbai, India \\ nishasarvadedvjti.org.in
}

\begin{abstract}
Nano machines can be connected together in a nano network. Molecular communication provides the most practical way in which nano machines can communicate with each other. This paper presents a review of pioneering research work in mathematical modelling and channel characterization of molecular communication for nano networks. It is reported that propagation of molecules can be modelled as deterministic as well as stochastic processes. Channel performance metrics like channel capacity, mutual information, gain/delay etc. have been estimated by various research groups. However, these parameters must be validated by evaluation of physical systems. Certain challenging issues like Brownian motion with negative drift, synchronization and inter-symbol interference in molecular channel are still open for investigation. Functionalities of higher network layers like modulation, error correction, routing etc. are yet to be exploited.
\end{abstract}

\section{KEYWORDS}

Nano Networks, Molecular Communication, Diffusion channel, Channel capacity

\section{INTRODUCTION}

Nanotechnology is an upcoming domain which promises revolutionary solutions to problems in interdisciplinary areas such as healthcare, agriculture, and environment, military, industrial and IT. All physical properties of nano scale materials show distinct variations with respect to the properties of corresponding bulk materials. This behaviour is attributed to quantum effects [1]. For example, the color of a piece of gold changes to red and copper becomes transparent in the realms of nano scale. The primary objective of nanotechnology is to synthesize materials, devices and systems of pre-determined characteristics which are otherwise not possible to manufacture.

A nano machine is a basic functional unit of any nano system. It consists of nano scale components which are able to perform a specific task such as communication, computation, data storing, sensing and/or actuation at nano level [2]. Individual nano machine can perform very limited tasks owing to its limited size and simplicity. However, number of nano machines may be co-ordinated through a network to perform elaborate functions. Such networks are termed as "Nano networks". An exhaustive overview of nano networking concepts can be found in $[2,3]$.

Nano machines connected in nano network may communicate with each other using technologies like electromagnetic, acoustic, nano-mechanical or molecular. Molecular communication (MC) uses encoded molecules as information carriers as opposed to EM waves in EM communications or light waves in optical communication. Transport of molecules for communication can be subdivided into two classes- active and passive. Active transport includes use of molecular motors in fluidic medium and it can be used for short ranges ( $\mathrm{nm}$ to few $\mathrm{mm}$ ). Passive transport can be either by using calcium signalling or by diffusion. This type of transport can be used for long range communications ( $\mathrm{mm}$ to $\mathrm{km})[3,4,5]$. $\mathrm{MC}$ is stochastic in nature, exhibits a large

DOI : $10.5121 /$ vlsic.2013.4203 
International Journal of VLSI design \& Communication Systems (VLSICS) Vol.4, No.2, April 2013

delay and shorter range compared to telecommunication. However it is biocompatible, energy efficient and can be used in aqueous medium [4]. Also, MC transceivers, owing to their size, provide easy integration with nano machines. MC may be useful in applications where traditional communication cannot be applied. Intra-body monitoring, nano sensor networks for consumer and industrial products, measurement of environmental parameters are a few examples [6]. For these reasons MC appears to be the best technology for nano networks. A brief comparison of classical communication and molecular communication is presented in table1.

\begin{tabular}{||l|c|c||}
\hline & TRADITIONAL & MOLECULAR \\
\hline CARRIER & Electromagnetic waves & Molecules \\
\hline DEVICES & Electronic & Nano machines \\
\hline SIGNAL & Electronic/ Optical & Chemical \\
\hline MEDIA & Space, Cables & Aqueous \\
\hline PROPAGATION SPEED & $\begin{array}{c}\text { Speed of light } \\
\left(3 \times 10^{8} \mathrm{~m} / \mathrm{s}\right)\end{array}$ & $\begin{array}{c}\text { Extremely low } \\
(\mathrm{nm}-\mu \mathrm{m} / \mathrm{s})\end{array}$ \\
\hline RANGE & Long distance $(\mathrm{m}-\mathrm{km})$ & Short distance $(\mathrm{nm}-\mathrm{mm})$ \\
\hline ENERGY CONSUMPTION & High & Low \\
\hline $\begin{array}{l}\text { MEDIUM } \\
\text { CONDITION }\end{array}$ & $\begin{array}{l}\text { Wired : Almost immune } \\
\text { Wireless : Affects comm. }\end{array}$ & $\begin{array}{c}\text { Affects communication } \\
\text { INFORMATION }\end{array}$ \\
\hline NOISE & Text, video, voice & $\begin{array}{c}\text { Phenomenon, chemical states, } \\
\text { processes }\end{array}$ \\
\hline \hline
\end{tabular}

Table 1: Comparison of traditional and molecular communication $[2,3,4]$

Shannon's communication theory defined the framework for contemporary communication. He introduced the notions of amount of information (entropy), channel capacity and various channel models for communication. Similar information theoretic problems are needed to be defined for $\mathrm{MC}$ before it is being put into practice. Currently various research groups are working towards mathematical modelling of MC channels. This includes accurately defining molecular transport in the channel, measuring performance metrics like channel capacity, delay, noise, suggesting link layer functions like modulation/demodulation, error correction for improved channel performance etc. However, direct application of information theoretic tools for MC will not be practical for following reasons:

- In classical communication, noise or attenuation is external to the flow of information. In MC noise is inherently present in the way information is propagated. i.e. randomness in diffusion process.

- The molecules released in the medium, linger in the channel for a long time. This property is referred to as the "memory" of molecular channel.

- Molecular signal transmission and reception processes tend to be very slow relative to traditional communication. This puts serious constraint on frequency of the channel use.

This paper is a survey of the literature directed towards characterization of molecular channels. For sake of brevity we will consider MC using drift-diffusion only. Rest of the paper is organized as follows: Section 2 discusses the mechanism of propagation of molecules. In section 3, molecular channel characterization using various parameters is described. Concluding remarks and open issues in the field are presented in section 4. 


\section{Propagation Dynamics}

As per the communication model described for MC in [7], a transmitter emits encoded message molecules according to molecular flux (difference in concentration of molecules) in the surrounding medium. These molecules will propagate through the medium by means of spontaneous diffusion. Some of these molecules will hit receiver which captures these molecules by its chemical receptors and decodes the message.

Messenger molecules

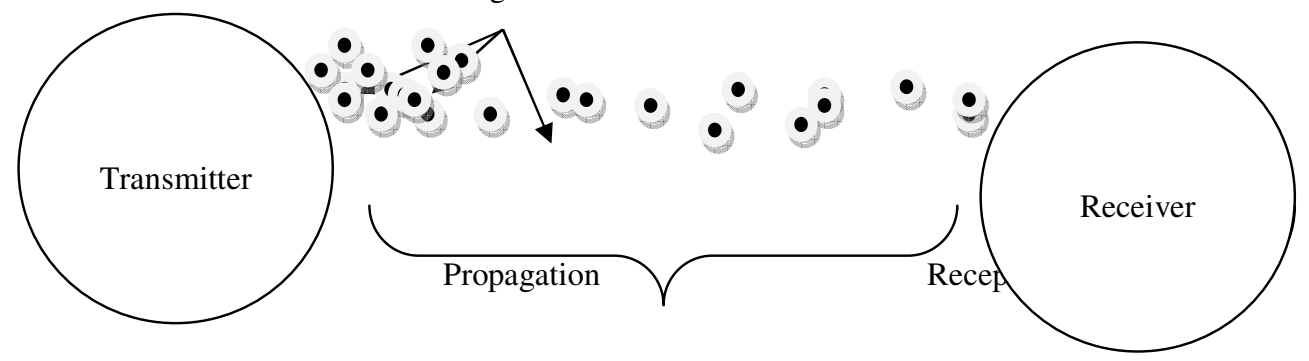

Fig. 1 Physical model of Molecular communication by diffusion

It is required that the medium through which the messenger molecules are moving is fluidic (air or water) for molecules to diffuse through it. MC is modelled as transmitter (emission of molecules), channel (diffusion through medium) and receiver (decoding of message) processes in [7, 8]. In certain cases, where the medium itself has a flow of its own, molecular motion is controlled by diffusion as well as the flow of the medium (drift). For example, movement of hormones through blood stream inside the human body. Also, external factors like turbulence created by flowing medium and antagonist agents must be accounted for [9].

There are two approaches for representing motion of molecules as mathematical models. One is by using Brownian motion (BM) and another is using Fick's laws of diffusion.

$\mathrm{BM}$ occurs due to random collisions and interactions of the molecules with the surrounding molecules (of the environment). It can be modelled as a continuous stochastic process. A detailed explanation of BM and random walk processes in general can be found in [10]. Propagation of molecules is characterized using BM in $[11,12,13,14]$.

The Probability Density Function for BM as a Wiener process shown in equation 2.1 is described in [12].

$$
f(w, x)=\frac{1}{\sqrt{2 \pi \sigma^{2} x}} \exp \left(-\frac{(w-v x)^{2}}{2 \sigma^{2} x}\right)
$$

Above expression gives position $w$ of the particle at time $x$ and is Gaussian with mean $v x$ and variance $\sigma^{2} x$.

A special case of BM is BM with drift. This represents motion of particles in a medium which has a definite flow. In equation 2.1 , the parameter $v$ represents drift velocity. References $[12,13]$ have incorporated positive drift velocities in their propagation models. However, negative drift velocity issue has not been addressed so far. To make a two-way communication between nano machines possible, positive as well as negative drift velocities must be taken care of.

The second approach for propagation modelling is deterministic which computes the position of a molecule with the help of differential equations defined by Fick's laws of diffusion which relate the flux of particles with the concentration gradient. Pierobon and Akyildiz have used this methodology in $[8,15]$. However, Fick's second law of diffusion needs a correction to take care of super-luminal propagation speed. Pierobon and Akilydiz have applied a telegraph equation to model the propagation of molecules in [8]. 
$\mathrm{BM}$ is a motion of individual particle whereas diffusion occurs at the macro scale. Garralda et al have used N3Sim simulator in [16] which simulates propagation of individual molecule using BM and verifies the results using Fick's Laws. Yet another attempt to combine deterministic and stochastic approaches can be found in [17] where propagation is modelled as diffusion followed by particle location displacement which is stochastic by nature.

Propagation due to only diffusion is a very slow process and may not be very useful for a meaningful communication. Therefore, a certain amount of drift needs to be introduced [9]. Fig. 2 shows effect of drift and diffusion on the propagation of molecular pulse. The spread observed in the shape of the pulse is due to diffusion and introduces an inter symbol interference (ISI) in MC.

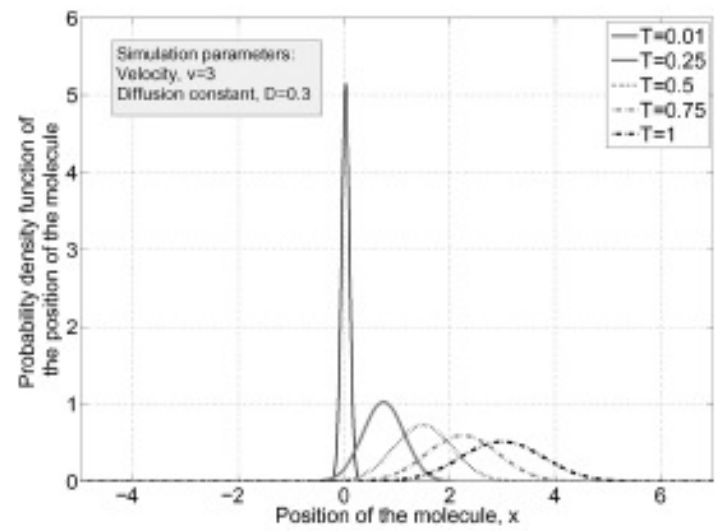

Fig. 2 Simulation results for PDF position for molecules at different times. Change in position is due to drift while change in variance is due to diffusion [12]

Miorandi has proposed [18] a stochastic model which takes into account various types of interactions of information molecules and environment molecules for the first time.

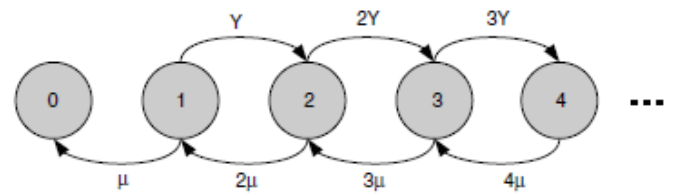

Fig. 3 Evolution diagram of number of molecules as Birth and Death process [18]

In this paper, the evolution of number of molecules in a communication channel over a specified channel is modelled as a birth-and-death process [10]. The interactions considered (absorption, generation and spontaneous emission) have different probabilities of occurrence. Spontaneous emission is considered to be a Poisson process.

\section{Channel Characterization}

MC forms a physical layer for the nano networks. However for accurate channel characterization various parameters like channel capacity, gain and delay must be measured. To improve upon these parameters link layer functions like modulation and error correction need to be defined.

\subsection{Channel parameters}

Capacity of a channel specifies maximum rate of transmission between the information source and the destination and is the most important channel parameter. As described by Shannon in [19], the channel capacity (C) can be computed as 
International Journal of VLSI design \& Communication Systems (VLSICS) Vol.4, No.2, April 2013

$$
C=\max \{I(X ; Y)\}
$$

Where $\mathrm{I}(\mathrm{X} ; \mathrm{Y})$ is a mutual information between transmitted signal $\mathrm{X}$ and received signal $\mathrm{Y}$. Eckford has computed mutual information as Monte Carlo estimation of PDF of the particle propagation [11]. By this method, transmitter is allowed to transmit one particle per slot. Kadloor et al have expressed mutual information as a concave function of input distribution [12]. To maximize the mutual information, input distribution should be optimized. The authors have solved this optimization problem using Blahut-Arimoto algorithm.

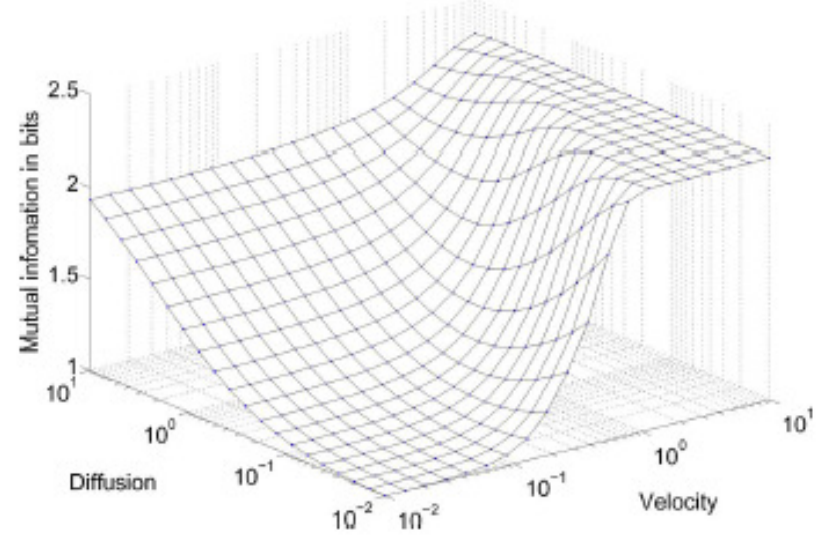

Fig.4 Grid plot indicating MI for various velocities and diffusion constants [12]

Figure 4 depicts the MI in bits with respect to diffusion constant and the velocity (i.e. drift). The velocity-diffusion region can be roughly classified into three regimes:

- A diffusion dominated region, where MI is relatively insensitive to the velocity

- A high-velocity region where MI is insensitive to the diffusion constant

- An intermediate regime, where the MI is highly sensitive to the velocity and diffusion constant of the medium

As expected MI increases with increase in velocity, and reaches a maximum value of $\log _{2}(\mathrm{~N})$ where $\mathrm{N}$ denotes number of slots are available for transmission. If a transmitter is allowed NOT to transmit a molecule in any particular slot then maximum MI is $\log _{2}(N+1)$. At high velocities, diffusion constant of the MI is insensitive to changes in diffusion constant.

The diffusion constant is a measure of the uncertainty in the propagation time. Hence, we would expect the MI to be lower when the diffusion constant is high. This is indeed the case at high velocities. However, it is surprising that a higher diffusion constant results in higher mutual information at low velocities. This is because, at low velocities, it is the diffusion in the medium which aids the propagation of the molecule from the transmitter towards the receiver. To improve MI at low velocity and low diffusion constants release of multiple molecules at a time is suggested.

Yet another approach in computing channel capacity is reported in [13], where diffusion channel is shown to be an Additive Inverse Gaussian Noise (AIGN) channel. Using differential entropy and additivity property of the IG distribution, channel capacity is estimated. Numerical results show that the mutual information is a complex function of drift velocity and diffusion coefficient. Nakano et al have considered molecule life expectancy (modelled as Poisson process) in computing the channel capacity [14]. Their results show that there is an optimal life expectancy that maximizes the channel capacity. 
Mutual information in diffusion based MC system is a function of bandwidth of transmitted signal, time interval for which particle concentration is considered (sampling time), diffusion coefficient of the medium, distance between transmitter and receiver and radius of the spherical receiver [17]. Also, numerical results of the same paper show that a data rate of few kbps can be achieved within a distance range of tenth of micrometer and for an average transmitted power around $1 \mathrm{pW}$.

Atakan and Akan have presented channel capacities for point-to-point, broadcast and multiple access channels in [20]. A book chapter [21] written by same authors computes channel capacity by using a channel model similar to binary symmetric channel and measures the effect of binding rate, temperature, bit transmission probability etc. on channel capacity.

Most of the papers mentioned above have considered timing channels i.e. information is embedded in transmission time of the molecules. Such channels require perfect synchronization in transmitter and receiver. The techniques presented in these papers must be suitably modified if there is a lack of synchronization. Moreover, there is a strong possibility of Inter symbol interference as explained in section 2. ISI restricts the transmission speed. It is required to develop coding techniques to avoid the effects of ISI.

Apart from channel capacity other parameters like gain and delay are also investigated. In [8], the authors have computed normalized gain and delay of a MC system by devising an equivalent electrical circuit for transmitter, channel and receiver.

\subsection{Simulations}

Although there are not many simulators available for diffusion based MC, a summary of useful tools is presented in [22]. N3Sim is a physical simulation framework for MC developed by Nanonetworking centre in Catalunya (N3Cat). This software is freely available at www.n3cat.upc.edu/n3sim.

Some of the important investigations (using N3Sim) in channel characterization for diffusion based MC have been reported. Llaster et al have proposed a pulse-based modulation scheme and derived various metrics like pulse width, delay and amplitude. Their results have been validated by N3Sim. An important finding of this research work is the pulse width is found to be inversely proportional to the diffusion coefficient of the medium. Therefore, the higher the diffusion coefficient the narrower the received pulses will be and in turn, may provide higher data rate in case of timing channels [23]. 
International Journal of VLSI design \& Communication Systems (VLSICS) Vol.4, No.2, April 2013

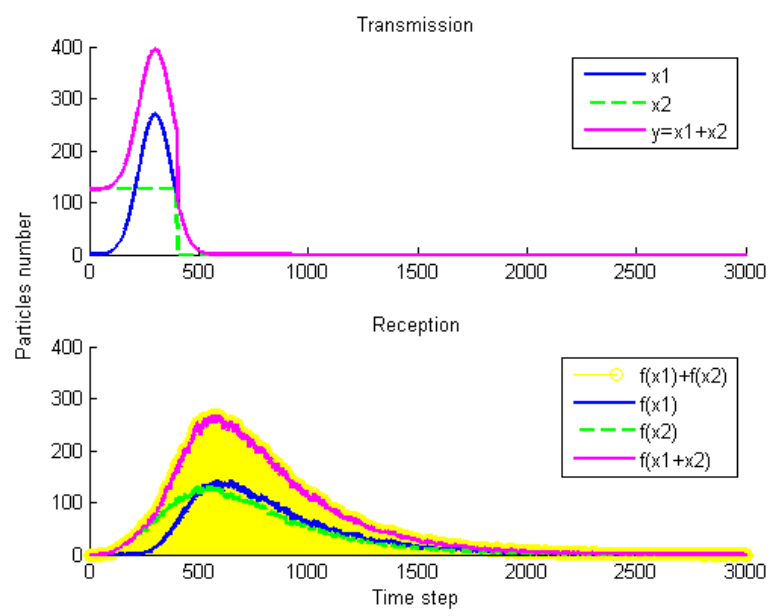

Fig.4 Transmission and reception of two pulses and their addition to prove linearity of molecular channel [16]

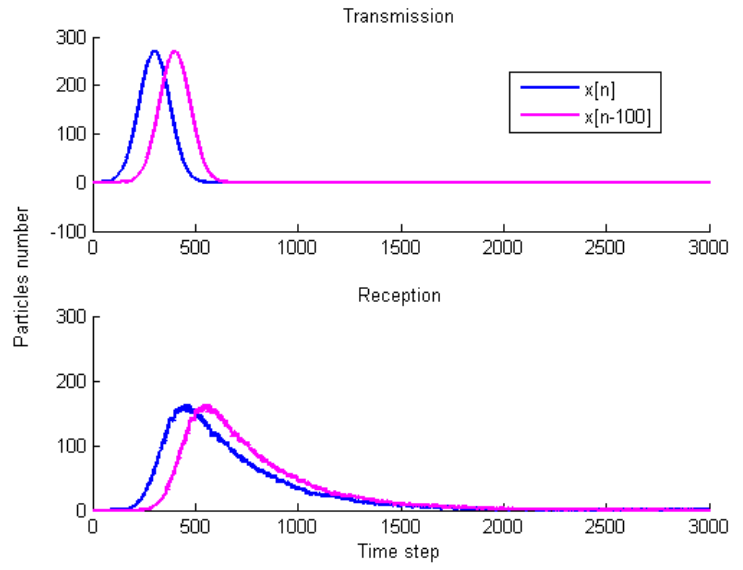

Fig.5 Transmission and reception of a single pulse and its time shifted version to prove temporal invariance of molecular channel [16]

Garralda et al have shown in [16] that a diffusion based MC channel in a linear time invariant channel, in single as well as multi transmitter scenarios. It is very clear from figure 4 that the superposition principle holds true in the example of 2 different pulses as well as their addition in a diffusive channel. This proves the linearity of molecular channel. Figure 5 shows that the timing relation between two signals remains constant through the diffusion based channel. 

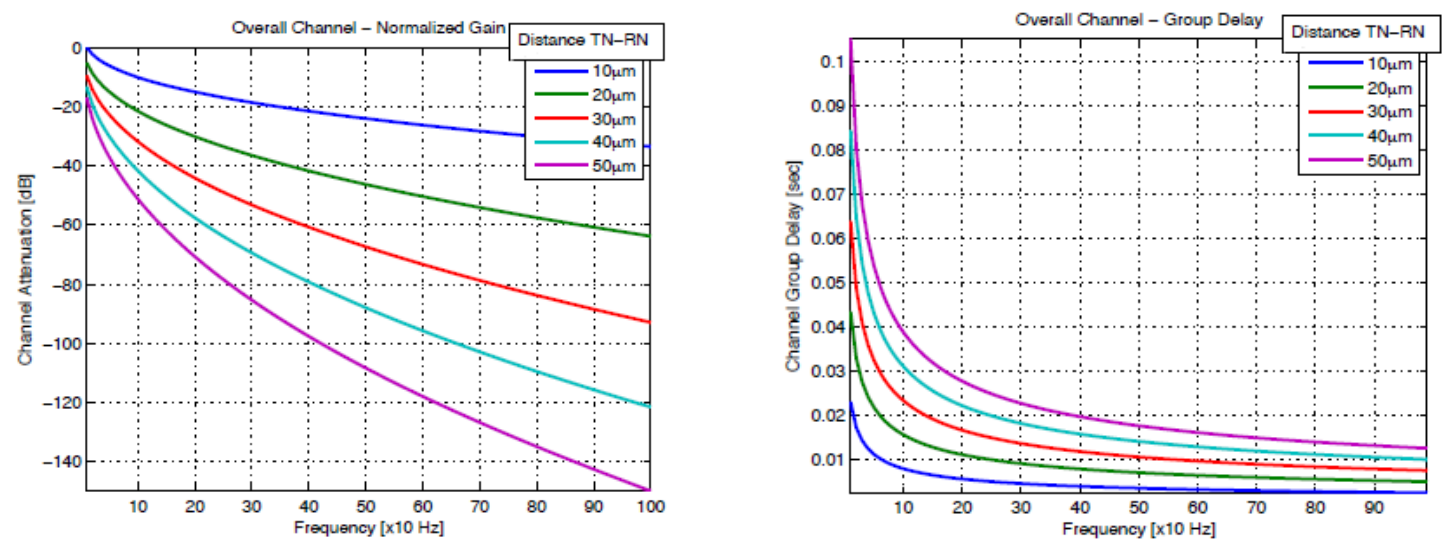

Fig. 6 a) Frequency response b) Group delay of molecular channel [8]

Pierobon et al have performed end-to-end analysis of channel gain and group delay in [8].

Figure 6 a) and b) show frequency response and group delay of molecular channel for various values of distance between transmitter and receiver. The curves are obtained using equivalent models for emission, diffusion and reception process explained in the same paper. Frequency spectrum considered here is $0-1 \mathrm{KHz}$ for diffusion coefficient $\mathrm{D}=10^{-6} \mathrm{~m}^{2} / \mathrm{s}^{2}$ for a Ca molecule diffusing in biological environment (Cellular cytoplasm).

The curves for the normalized gain in Fig. 6 a) show the maximum value $1(0 \mathrm{~dB})$ at the frequency $0 \mathrm{~Hz}$ and they monotonically decrease as the frequency increases and approaches $1 \mathrm{kHz}$. This phenomenon can be explained considering that if the frequency of the end-to-end model input signal increases, the resulting modulated particle concentration rate decreases in its magnitude. This is due to the fact that the particle mobility in the diffusion process between the inside and the outside of the transmitter is constrained by the diffusion coefficient. The higher is the diffusion coefficient, the faster is the diffusion process given a value for the particle concentration gradient between the inside and the outside of the transmitter.

The curves for the normalized group delay in Fig. 6 b) show nonlinear behaviour with respect to frequency as well as with respect to distance between transmitter and receiver. Therefore, the channel is supposed to exhibit a dispersive behaviour in the frequency range from $0 \mathrm{~Hz}$ to $1 \mathrm{kHz}$ and, consequently, the signal propagating through the molecular diffusion module can be distorted.

Lacasa has performed a similar analysis in [9] and observed the frequency dispersion behaviour; She has suggested a "chirp" function to be used for signal transmission in MC. The chirp is a frequency variation in time and, as channel's behavior happens to have dispersion, this would compensate for the distortion in frequency as time and space vary. Therefore, the transmission of a chirped pulse may be able to equalize the channel effect.

A short comparison of the research works in MC channel characterization is summarized in table. 
International Journal of VLSI design \& Communication Systems (VLSICS) Vol.4, No.2, April 2013

1.

\begin{tabular}{|c|c|c|c|c|c|}
\hline & $\begin{array}{c}\text { Propagation } \\
\text { Model }\end{array}$ & $\begin{array}{c}\text { Channel } \\
\text { Parameters }\end{array}$ & Modulation & $\begin{array}{c}\text { Major } \\
\text { Contributions } \\
\end{array}$ & Future scope \\
\hline $\begin{array}{l}\text { Pierobon } \\
2010[8]\end{array}$ & $\begin{array}{l}\text { Fick's laws with } \\
\text { telegraph } \\
\text { equation }\end{array}$ & NG, ND & ------------- & $\begin{array}{l}\text { Equivalent electric } \\
\text { circuits developed for } \\
\text { MC model }\end{array}$ & ------------ \\
\hline $\begin{array}{l}\text { Eckford } \\
2007[11]\end{array}$ & Brownian motion & $\mathrm{CC}$ & Timing & $\begin{array}{l}\text { Preliminary propagation } \\
\text { and noise } \\
\text { characterization } \\
\text { presented }\end{array}$ & $\begin{array}{l}\text { Transmission } \\
\text { rate constraints, } \\
\text { Input } \\
\text { distributions } \\
\text { optimization }\end{array}$ \\
\hline $\begin{array}{l}\text { Kadloor } \\
2012 \text { [12] }\end{array}$ & $\begin{array}{l}\text { Brownian motion } \\
\text { with positive } \\
\text { drift velocity }\end{array}$ & $\mathrm{CC}$ & PPM, PAM & $\begin{array}{l}\text { BM with positive drift } \\
\text { considered for first time } \\
\text { MI's dependence on } \\
\text { velocity and diffusion } \\
\text { established }\end{array}$ & $\begin{array}{l}\text { BM with } \\
\text { negative drift } \\
\text { Channel } \\
\text { estimation for } \\
\text { unknown drift } \\
\text { velocity }\end{array}$ \\
\hline $\begin{array}{l}\text { Srinivas } \\
\text { 2012[13] }\end{array}$ & $\begin{array}{l}\text { Brownian motion } \\
\text { with positive } \\
\text { drift velocity }\end{array}$ & $\mathrm{CC}$ & Timing & $\begin{array}{l}\text { MC channel shown to } \\
\text { be AIGN channel } \\
\text { MI's dependence on } \\
\text { velocity and diffusion } \\
\text { established }\end{array}$ & $\begin{array}{l}\text { ISI mediation, } \\
\text { Synchronizatio } \\
\mathrm{n} \text {, } \\
\text { Amplitude } \\
\text { modulation }\end{array}$ \\
\hline $\begin{array}{l}\text { Nakano } \\
2012[14]\end{array}$ & Brownian motion & $\mathrm{CC}$ & BCS & $\begin{array}{l}\text { CC's dependence on } \\
\text { molecular life } \\
\text { expectancy established, } \\
\text { Multiple bits can be } \\
\text { transmitted in single slot }\end{array}$ & $\begin{array}{l}\text { Transmission } \\
\text { of multiple bits, } \\
\text { Error } \\
\text { correction, } \\
\text { Multiple } \\
\text { transmitters }\end{array}$ \\
\hline $\begin{array}{l}\text { Pierobon } \\
2010[15]\end{array}$ & Fick's laws & |-------------- & -------------- & $\begin{array}{l}\text { Physical and stochastic } \\
\text { models for noise } \\
\text { developed }\end{array}$ & --------------- \\
\hline $\begin{array}{l}\text { Garallda } \\
\text { 2011[16] }\end{array}$ & $\begin{array}{l}\text { Brownian motion } \\
\text { + Fick's laws }\end{array}$ & NG,ND & $\begin{array}{l}\text { PAM,PPM, } \\
\text { CtS, RM }\end{array}$ & $\begin{array}{l}\text { MC channel shown to } \\
\text { be LTI channel } \\
\text { OOK found to be best } \\
\text { modulation scheme } \\
\text { Spike is optimal pulse } \\
\text { shape }\end{array}$ & $\begin{array}{l}\text { Data rate, } \\
\text { channel } \\
\text { capacity needs } \\
\text { to be estimated }\end{array}$ \\
\hline $\begin{array}{l}\text { Pierobon } \\
\text { 2013[17] }\end{array}$ & $\begin{array}{l}\text { Fick's laws and } \\
\text { particle location } \\
\text { displacement }\end{array}$ & $\mathrm{CC}$ & --------------- & $\begin{array}{l}\text { Average transmission } \\
\text { power analyzed } \\
\text { CC expressed in terms } \\
\text { of ATP and BW } \\
\text { CC not dependent on } \\
\text { specific coding scheme }\end{array}$ & $\begin{array}{l}\text { More stringent } \\
\text { upper bounds }\end{array}$ \\
\hline $\begin{array}{l}\text { Miorandi } \\
2011[18]\end{array}$ & $\begin{array}{l}\text { Birth and Death } \\
\text { Process } \\
\text { (flow based) } \\
\text { Brownian } \\
\text { motion } \\
\text { (diffusion based) } \\
\end{array}$ & -------------- & --------------- & $\begin{array}{l}\text { Interaction of message } \\
\text { molecules with other } \\
\text { molecules considered } \\
\text { for first time }\end{array}$ & $\begin{array}{l}\text { Optimal } \\
\text { coding/ } \\
\text { detection } \\
\text { schemes }\end{array}$ \\
\hline $\begin{array}{l}\text { Atakan } \\
2010[20]\end{array}$ & Fick's laws & $\mathrm{CC}$ & BCS & $\begin{array}{l}\text { CC for poit-to-point, } \\
\text { broadcast and multiple } \\
\text { access channels } \\
\text { analyzed }\end{array}$ & $\begin{array}{l}\text { Routing } \\
\text { scheme for } \\
\text { multiple access } \\
\text { systems }\end{array}$ \\
\hline $\begin{array}{l}\text { Atakan } \\
2008[21]\end{array}$ & $\begin{array}{l}\text { Ligand-binding } \\
\text { receiver model }\end{array}$ & ---------' & BCS & $\begin{array}{l}\text { Effect of environmental } \\
\text { factors on CC measured, } \\
\text { Molecular Error } \\
\text { compensation scheme } \\
\text { used ( more than } 100 \% \\
\text { capacity improvement) }\end{array}$ & ------------- \\
\hline
\end{tabular}

Table 1 Summary of MC channel characterization 
International Journal of VLSI design \& Communication Systems (VLSICS) Vol.4, No.2, April 2013

LEGENDS: $\mathrm{NG}=$ Normalized gain ND $=$ Normalized delay $\mathrm{CC}=$ Channel Capacity $\mathrm{BCS}=$ Binary Coding System $\mathrm{MI}=$ Mutual Information ATP= Average Thermodynamic Power

\section{Conclusions}

Nano networking is a novel idea which will extend the functionalities of nano machines. Molecular communication is the most feasible way in which nano machines can communicate with each other. However, MC has still a long way to go in terms of mathematical modelling and channel characterization. This paper has presented a review of the current research work in this area. It is found that propagation of molecules can be modelled as deterministic as well as stochastic processes. Moreover, these two approaches can be combined for getting accurate results. Some important characteristics of molecular channels are - Non-linear frequency response giving rise to distortion in transmitted signal, Delay varies non-linearly with frequency and distance between transmitter and receiver, channel exhibits Inter-symbol Interference (ISI), If drift is present, the channel can be modelled as Additive Inverse Gaussian channel.

Channel performance metrics like channel capacity, mutual information, gain/delay etc. have been estimated by various research groups. However, these are theoretical models and physical models are needed to be developed for stringent evaluation of channel parameters.

Certain challenging issues like BM with negative drift are still open for investigation. Functionalities of higher network layers like modulation, error correction, routing etc. are yet to be exploited.

\section{REFERENCES}

[1] M. Dragoman, D. Dragoman, (2009) "Nanoelctronics: Principles and Devices", Boston, London, Artech House

[2] I.F.Akyildiz, F. Brunetti, C. Blázquez , (2008) "Nanonetworks: A New Communication Paradigm," Computer Networks, Vol.52, Elsevier, pp 2260-2279

[3] T. Nakano, M.Moore, F.Wei, A. Vasilakos and J. Shuai, (2012) "Molecular Communication and Networking: Opportunities and Challenges", IEEE Transactions on Nanobioscience, Vol.11, No.2, pp $135-148$

[4] T. Nakano, M.Moore, A.Enomoto and T. Suda, (2011) "Molecular Communication as Biological ICT", in Biological Functions for Information and Communication Technologies, Vol. 320, H. Sawai Ed. , Springer, pp 49-86

[5] L.P.Giné, I.F.Akyildiz,(2009)“Molecular Communication for Long Range”, Computer Networks, Vol.53, Elsevier, pp 2753-2766

[6] S. Hiyama, Y. Moritani, T. Suda, (2008)“Molecular Transport System in Molecular Communication”, NTT DOCOMO Technical Journal, Vol. 10, No.3, pp.49-53, 2008

[7] I.F. Akyildiz, J.M. Jornet, M. Pierobon, (2010)“Propagation Models for Nanocommunication Networks", IEEE Proceedings of EuCAP

[8] M. Pierobon, I.F. Akyildiz, (2010) "Physical End-to-End Model for Molecular Communication in Nanonetworks", IEEE Journal on Small Areas in Communications, Vol. 28, No. 4, pp. 602-611

[9] N.R.Lacasa, (2009) "Modelling Molecular Communications Networks", M.S. Thesis, Elect. and Comp. Engg. Dept. Georgia Tech. Univ., GA

[10] S.M. Ross, (2001) "Brownian Motion and Stationary Processes" in "Introduction to Probability Models", 7th ed., Academic Press, Harcourt India, ch.10, sec. 10.3.1, pp.554-555

[11] A. W. Eckford, "Nanoscale Communication with Brownian Motion", in Proc 41st Annual Conference of Information Sciences and Systems CISS'07, pp 160-165

[12] S. Kadloor, R. S. Adve, A. W. Eckford, (2012) “ Molecular Communication Using Brownian Motion with Drift”, IEEE Transactions On Nanobioscience, Vol. 11, No. 2, pp.89-99

[13] K.V. Srinivas, A. W. Eckford, R. S. Adve, (2012)“ Molecular Communication in Fluid Media: The Additive Inverse Gaussian Noise Channel”, IEEE Transactions On Information Theory, Vol. 58, No. 7, pp. 4678-4692 
International Journal of VLSI design \& Communication Systems (VLSICS) Vol.4, No.2, April 2013

[14] T. Nakano, Y. Okaie, J.Q. Liu, (2012) "Channel Model and Capacity Analysis of Molecular Communication with Brownian Motion", IEEE Communications Letters, Vol. 16, No. 6, pp. 797-800

[15] M. Pierobon, I.F. Akyildiz (2010)“Diffusion-based Noise Analysis for Molecular Communication in Nanonetworks", IEEE Journal on Small Areas in Communications, Vol. 28, No. 4, pp. 602-611

[16] N. Garralda , I. Llatser, A. Cabellos-Aparicio , E. Alarcón, M. Pierobon, (2011) "Diffusion-based physical channel identification in molecular nanonetworks", Nano Communication Networks, Vol. 2 , Issue 4, pp. 196-204

[17] M. Pierobon, I.F. Akyildiz (2013) "Capacity of Diffusion Based Molecular Communication System with Channel Memory and Molecular Noise”, IEEE Transactions on Information Theory, Vol. 59, No. 2, pp. 942-954

[18] D. Miorandi, (2011) “A stochastic model for Molecular Communication”, Nano Communication Networks, Vol. 2, Issue 4, pp. 196-204

[19] C. E. Shannon, (1948) "A mathematical theory of Communication," The Bell System Technical Journal, vol. 27, pp. 379-423

[20] B. Atakan, O. Akan, (2010) "Deterministic Capacity of Information Flow in Molecular Nanonetworks", Nano Communication Networks, Vol. 1, Issue 1, pp. 31-42

[21] B. Atakan, O. Akan, (2008) "On Channel Capacity and Error Compensation in Molecular Communication", in Transactions on Computational Systems Biology, pp.59-80, Springer-Verlag Berlin, Heidelberg,

[22] S.F. Bush, (2010) "Nanaoscale Communication Networks", Boston, London, Artech House,

[23] I. Llatser, E. Alarcon, M. Pierobon, (2011) "Diffusion Based Channel Characterization in Molecular Nanonetworks," IEEE International Workshop on Molecular and Nano Scale Communication (MoNaCom)

\section{Authors}

Prachi Raut received B.E. and M.E. degrees in Electronics Engineering from Mumbai University. She is working as an Associate Professor at St. Francis Institute of Technology, Mumbai, India. Currently, she is pursuing PhD under guidance of Dr. Nisha Sarwade at the VJTI, Mumbai, India. Her areas of specialization are Nano networks, Convergence Technologies and Network security.

Dr. Nisha Sarwade received B.E. degree in Electronics Engineering from Jiwaji University, Gwalior and M.E. (Solid State Electronics) and $\mathrm{PhD}$ (Electronics Engineering) from University of Roorkee. She was working as a lecturer at the University of Roorkee during 1983-1987. Currently she is working as an Associate Professor at the VJTI, Mumbai, India. Her research interests include Nano Electronics with emphasis on CNT, Compund semiconductors, High-k dielectrics and flash memories and Microwave circuit design. She has 56 national as well as international

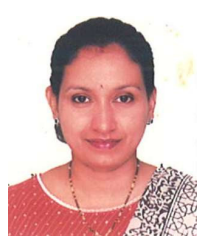
publications to her credit. 